\title{
EXPRESSION OF ESTROGEN RECEPTOR $\beta$ ISOFORMS IS REGULATED BY TRANSCRIPTIONAL AND POST-TRANSCRIPTIONAL MECHANISMS
}

\section{Laura Smith, Louise J Coleman, Michele Cummings, Sampoorna Satheesha, Spencer O Shaw, Valerie Speirs, and Thomas A Hughes}

Leeds Institute of Molecular Medicine, Leeds University, Leeds, LS9 7TF, UK

Short title: Regulation of ER $\beta$ isoform expression

Correspondence to: Thomas A Hughes, Leeds Institute of Molecular Medicine, St. James's 


\section{SYNOPSIS}

Although estrogen receptors (ERs) mediate breast tumour behaviour, the precise role of ERß remains unclear. This is mainly because analyses have been complicated by the presence in breast tissue of three ERß protein variants (ERß1, 2 and 5) that derive from differential 3' splicing. We have recently identified the first known mechanisms responsible for the differential control of isoform expression, involving regulation of translation via 5 , untranslated regions (UTRs). In this study, we have uncovered further complexity involving the influence of multiple promoters and cross-talk between 5' and ' 3 'UTRs.

We demonstrate that full-length ERß messages are transcribed from three separate promoters; two promoters are well-established within the literature while the third represents a novel finding. Each promoter produces transcripts with distinct 5'UTRs. The differential 3' splicing that produces transcripts coding for the ER $\beta$ isoforms also defines isoform-specific 3'UTRs. We identified exact 3'UTR sequences for each isoform, and show that alternative polyadenylation sites are used in a cell-type specific manner to produce transcripts with 3'UTRs of different lengths. Critically, we show that 5' and 3'UTRs combine to specify the efficiencies with which individual transcripts are translated, with 3'UTR length having a key influence. In addition, we demonstrate how 17ß-estradiol, a key driver of breast cancer development, impacts on the regulation of ERß expression at both transcriptional and translational levels.

Keywords: untranslated regions; translational regulation; UTRs; differential polyadenylation; esr2 


\section{INTRODUCTION}

Estrogen receptors (ERs) are critical mediators of estrogen function and play roles in many pathological processes, especially carcinogenesis [1]. Roles of ER $\alpha$ are relatively well understood while those of ER $\beta$ remain unclear. This is due, in part, to a reported discrepancy between ER $\beta$ expression at mRNA and protein levels, leading to conflicting expression data [2, 3]. Also, ER $\beta$ is expressed as at least five protein isoforms, derived from differential 3 ' splicing of ER $\beta$ transcripts [4], yet their potential to have distinct functions has often been ignored in favour of analysis of total ER $\beta$. In breast tissue, ER $\beta 1,2$ and 5 predominate, with each appearing to have separate biological functions as demonstrated by their associations with different breast cancer types and prognoses [5-8]. Differences between the isoforms are also evident in terms of comparisons of expression in normal breast tissue and breast cancer. ER $\beta 1$ is frequently downregulated in cancer compared with normal cells $[9,10]$ suggesting that it may function as a tumour suppressor [11-13]. However, ER $\beta 2$ appears to be up-regulated during carcinogenesis $[10,14]$, while ER $\beta 5$ may also be up-regulated, at least at the level of mRNA [15]. Little is known about the mechanisms responsible for these changes in ER $\beta$ expression. ER $\beta$ promoter methylation has frequently been observed in breast cancers, and this is thought to be responsible for down-regulation of some ER $\beta$ transcripts, although this appears to be at odds with the reported up-regulation of ER $\beta 2$ and 5. We have recently identified the first known mechanisms responsible for differential control of expression of the different isoforms involving regulation of translation via two alternative 5' untranslated regions (UTRs) [16]. We have now extended this theme to examine thoroughly the regulatory functions of the extensive range of ER $\beta$ UTRs, and have determined further mechanisms for differential control of ER $\beta$ isoform expression.

\section{EXPERIMENTAL}

\section{Cell culture, transfection, flow-cytometry and dual luciferase assays}

Cell lines representing breast cancers of luminal (MCF7) and basal (MDAMB-231) subtypes and benign non-transformed breast tissue (HB2) were obtained from the European Collection of Animal Cell Cultures. Cells were cultured in RPMI-1640 containing 5\% FCS (MCF7, MDAMB231 ) or DMEM containing or $10 \%$ FCS (HB2) (both Invitrogen, Paisley, UK) at $37^{\circ} \mathrm{C}$ in $5 \%$ $\mathrm{CO}_{2}$. Bi-monthly mycoplasma checks (MycoAlert® Mycoplasma detection assay, Lonza, USA) were consistently negative and STR profiles confirmed cell identity. Cells were transfected as previously described [16]. For experiments using exogenous 17 $\beta$-estradiol (E2), cells were cultured in phenol red free medium (Invitrogen, Paisley, UK) supplemented with charcoalstripped FCS. 5h post-transfection, fresh medium or medium containing 10nM E2 (SigmaAldrich, Poole, UK) was added before analysis after $24 \mathrm{~h}$. For flow-cytometry, cells were removed from wells with trypsin and resuspended in fresh medium containing $1 \%$ serum. GFP expression was quantified (mean fluorescent intensity of $10^{4}$ events after exclusion of debris/dead cells on the basis of forward activated light scatter vs side scatter) at $525 \mathrm{~nm}$ (LSRII, BD Biosciences, Oxford, UK). Gates were set so that $<1 \%$ of untransfected cells were defined as expressing GFP. Dual luciferase assays (Promega, Madison, WI, USA) were performed according to the manufacturer's instructions using a Lumat LB9507 luminometer (Berthold Technologies, Harpenden, UK). pSV40-renilla (Promega, Madison, WI, USA) was used as a control. 


\section{Plasmid construction}

pTH-GFPa and green fluorescent protein (GFP) reporters for UTRa and UTRc have been described previously $[16,17]$. The GFP reporter for the E1 5'UTR was cloned similarly to those for UTRa and UTRc; the UTR was amplified by PCR from cDNA prepared from MCF7 cells and was cloned upstream of the GFP ORF in pTH-GFPa. Importantly, this strategy removes the 5 ' end of the multiple cloning site, allowing the inserted 5'UTRs to be immediately adjacent to the transcriptional start site. 3'UTRs were amplified by PCR from cDNA prepared from HB2 cells and were cloned downstream of the GFP ORF in each of the 5'UTR reporter constructs with BamHI/HindIII. Promoter sequences were amplified from MCF7 genomic DNA and cloned into pGL3-Basic (Promega) using KpnI/NheI. The E1 promoter was cloned as two fragments: a 3' fragment cloned using KpnI/NheI then a 5' fragment cloned using KpnI. Primer sequences are listed in Table S1.

\section{cDNA synthesis and PCR}

RNA was purified from cells with RNeasy kits (Qiagen, Crawley, UK); contaminating DNA was removed with Turbo DNase I (Applied Biosystems, Warrington, UK). First strand cDNA was synthesized using SuperScript II (according to the manufacturer's protocol) and oligo(dT) or random hexamers. Triplicate real-time PCR analysis was performed (Applied Biosystems SYBR ${ }^{\circledR}$ Green PCR Master Mix and 7900HT machine). Dissociation curves and serial cDNA dilutions were performed to ensure primer specificities and equivalent amplification efficiencies; correlation coefficients of $>0.985$ and primer efficiencies of $>95 \%$ and $<100 \%$ were deemed acceptable. Reactions were also performed using template lacking reverse transcriptase (RT): products were either undetectable or greatly reduced $(>30,000$ fold less product than the equivalent $\mathrm{RT}+$ ) hence genomic or plasmid DNA contamination was not considered to interfere with data. Expression of UTRs was determined relative to expression of the RPLP0 (36B4) gene [18]. "Rapid amplification of cDNA ends" (RACE) was performed using 5'RACE System2 or 3'RACE System (both Invitrogen) according to the manufacturer's instructions (both standard and a modified adapter primer were used for ER 32 3'RACE). All primer sequences are listed in Table S1. Products were analyzed on $2.5 \%$ agarose $(0.5 \mu \mathrm{g} / \mathrm{ml}$ ethidium bromide, $1 \times \mathrm{TBE})$ and visualised on an UV trans-illuminator. Products were excised from gels and cloned into pGEMTeasy (Promega); at least five clones for each were sequenced. Note: products are larger than the UTRs they represent since they include some reading frame and the RACE adapters.

\section{$R N A$ structure and statistical analyses}

Modelling was performed using mfold v3.1 as previously to predict potential secondary structures for RNA molecules [16]. The algorithm finds base-pairing solutions that are sterically possible and release the greatest amount of free energy $(\Delta G)$ during structural folding; more stable structures release more energy as they form and therefore have greater $\Delta G$ values [19]. The Student's $t$-test was used for statistical analysis using Microsoft Excel. All P-values were two-sided; $\mathrm{p}<0.05$ was considered significant.

\section{RESULTS}

\section{A novel 5 'UTR for ERB}

We previously studied the regulatory roles of two ER $\beta$ 5'UTRs that we termed UTRa and UTRc [16]. These 5'UTRs result from transcription initiation from two alternative promoters and 
mutually exclusive splicing of the untranslated first exons, exon $0 \mathrm{~K}$ or $0 \mathrm{~N}$, to the first coding exon (exon 1) [20]. 5'RACE analyses were performed in breast cell lines in order to examine ER $\beta$ 5'UTRs further. Products representing 5'UTRs containing only sequence from exon 1 or from immediately upstream of the 5' splice site of exon 1 were the sole products obtained from primers located within the coding region of exon 1 (Fig 1A). UTRa or UTRc could only be detected in 5'RACE reactions using primers specific for their respective upstream exons, $0 \mathrm{~K}$ and ON [16]. RACE products containing only sequence from within the accepted bounds of exon 1 could be interpreted in two ways: as truncated versions of UTRa or UTRc, or as complete 5'UTRs derived from transcriptional initiation within exon 1. Products containing sequence upstream of the accepted bounds of exon 1 were likely to result from transcriptional initiation adjacent to exon 1. Fig 1B shows an alignment of mRNAs containing either UTRa, UTRc or the novel shorter 5'UTR ("UTR-E1") with the 5' end of the human ER $\beta$ gene on 14q23. The diagram depicts putative transcriptional initiation (bent black arrows) over a range of sequences upstream of and within exon 1 allowing expression of a 5' extension to exon 1 (grey box) on some UTR-E1 containing transcripts. We next performed qPCR to examine the relative expression of UTRa, UTRc and UTR-E1 in breast cell lines. We analysed expression of UTR-E1 using a primer that was complementary to sequence within this 5 ' extension of exon 1 , and was therefore not contained within UTRa or UTRc. It is worth noting that these analyses may underrepresent UTR-E1 expression since RACE analyses show that the 5' extension of exon 1 is present on only a subset of UTR-E1 containing transcripts. All three breast cell lines examined expressed UTR-E1 (Fig 1C). Products were not amplified from mock reverse transcription reactions providing validation that transcribed/reverse transcribed sequences were detected rather than contaminating genomic DNA. In HB2 and MCF7 cells, UTRa was the majority species, with UTRc and UTR-E1 being expressed at similar lesser levels. In contrast, MDAMB-231 cells expressed $\sim 12.5$-fold more UTRc, and $\sim 3.5$-fold more UTR-E1, than UTRa. We concluded that UTR-E1 represented a novel 5'UTR for ER $\beta$.

\section{A third transcriptional promoter for ERB}

Next, our hypothesis was that the DNA immediately upstream of exon 1 acts as a promoter allowing expression of transcripts containing UTR-E1. We cloned $\sim 2 \mathrm{~kb}$ of the genomic DNA directly upstream of exons $0 \mathrm{~K}, 0 \mathrm{~N}$ or $\mathrm{E} 1$ into separate luciferase reporter vectors and performed luciferase assays in $\mathrm{HB} 2$ and MCF7 breast cell lines in order to examine the relative promoter activities of the two known ER $\beta$ promoters and the putative third promoter. Activities are shown in each cell type (Fig 2). The putative third promoter ("promoter E1") showed activity in both cell lines. In HB2 cells, promoter E1 showed intermediate activity between promoters $0 \mathrm{~N}$, the most active, and $0 \mathrm{~K}$, the least. In MCF7 cells, promoters $0 \mathrm{~K}$ and $0 \mathrm{~N}$ determined similar transcriptional activities whilst promoter E1 was $\sim 70 \%$ weaker. We concluded that ER $\beta$ transcription is indeed driven by a third, previously uncharacterised, promoter that determines expression of UTR-E1.

\section{5'UTRs differentially regulate efficiency of ERß translation}

We have previously shown that UTRa and UTRc have profound and differential influences on ER $\beta$ translation. We hypothesized that UTR-E1 may also influence translation, thus we extended our previous analyses to test this using our established green fluorescent protein (GFP) reporter assay $[16,21]$. UTRa, UTRc and UTR-E1 were cloned upstream of the GFP reading frame in expression vectors. For UTR-E1, we cloned the sequence encoded by the published extent of 
exon 1, representing a commonly identified 5'RACE product. Cells were transiently transfected with equal numbers of copies of vectors to allow expression of GFP mRNAs either with nonregulatory 5'UTRs (positive control; “con”), or with UTRa, UTRc or UTR-E1. GFP protein expression was measured by flow-cytometry and GFP mRNA expression was measured by qPCR allowing determination of relative translational efficiencies for each GFP message (Fig 3). As previously published, UTRa and UTRc inhibited translation, with UTRa being strikingly inhibitory in nature. UTR-E1 was also inhibitory in nature despite being short (only 90 nucleotides; as compared to UTRa, 289, and UTRc, 418 nucleotides) and having a very low predicted degree of secondary structure (as assessed by the theoretical change in free energy of folding, $\Delta \mathrm{G}$; UTR-E1's predicted $\Delta \mathrm{G}$ is $-14 \mathrm{kcal} / \mathrm{mole}$ while, for comparison, those of UTRa and UTRc are -84 and $-166 \mathrm{kcal} / \mathrm{mole})$. A consistent pattern of relative influences for each UTR was seen in all cell lines, with UTRa being more, and UTR-E1 less inhibitory. We concluded that ER $\beta$ 5'UTRs specified the efficiencies with which downstream ORFs are translated. We also noted that promoter E1 may be especially important in terms of defining ER $\beta$ function since transcripts from this promoter, which contain UTR-E1, are the most efficiently translated ER $\beta$ mRNAs.

\section{Cross-talk between 5' and 3'UTRs influences translation of individual ERß isoforms}

Transcripts for at least three functionally-distinct ER $\beta$ isoforms are produced in breast cells [15]. These are derived from differential 3' splicing of the final ER $\beta$ exons [4]. It is evident that this differential splicing must also confer different 3'UTRs on transcripts for each isoform, although the exact 3'UTR sequences are poorly defined especially with respect to which potential polyadenylation sites are used. We were interested to examine whether ER $\beta$ 3'UTRs might influence translation. First, we performed 3'RACE analyses for each isoform in HB2 and MCF7 breast cells to identify 3'UTR sequences (Fig 4A). For ER $\beta 1$, we were only able to amplify a product in MCF7 cells, probably because ER $\beta 1$ expression levels were low in HB2 cells. The 3'UTR identified was of 242 nucleotides, representing a considerable 3' extension of the published sequence (108 nucleotides; accession NM_001437.2). For ER $\beta 2$ we identified three 3'UTRs of similar lengths: an 85 nucleotide sequence in HB2 cells and 103 or 108 nucleotide sequences in MCF7 cells; each apparently terminating at polyadenylation sites slightly more proximal than the published $3^{\prime}$ UTR sequence of 120 nucleotides (accession AF051428). Celltype specific alternative 3'UTRs were identified for ER $\beta 5$ : sequences of 234 nucleotides in HB2 and of 79 nucleotides in MCF7 cells. These represented use of either proximal or distal polyadenylation sites as compared to the published sequence of 177 nucleotides (accessions DQ838583.1 and AF061055.1). We did not detect expression of an extended ER $\beta 2$ 3'UTR that is represented within Genbank (accession NM_001040276.1; 498 nucleotides). However, RACE reactions can be biased towards amplification of shorter sequences, therefore we designed primers to assess expression of this specific 3'UTR. The longer ERß2 3'UTR (“ $\beta 2$-long”) was, in fact, expressed in both cell lines (Fig 4A; right hand panel). These data are summarised in Fig $4 \mathrm{~B}$ in the form of an alignment of the 3' ends of mRNAs for each isoform with the 3' end of the human ER $\beta$ gene.

We were interested to examine whether these isoform-specific 3'UTRs define different translational efficiencies in conjunction with the 5'UTRs, thereby allowing differential expression of the isoforms. We cloned 3'UTRs downstream of the GFP reading frame in each 5'UTR GFP reporter construct. For ER $\beta 2$, we focused on comparison of $\beta 2$-long, as detected by 
specific PCR, with the sequences identified using RACE (" $\beta 2$-short"; we examined a 120 nucleotide sequence, accession AF051428). For ER $\beta 5$ we examined both sequences identified by RACE (" $\beta 5$ HB" and " $\beta 5$ MCF”). HB2 and MCF7 cells were transiently transfected with equal numbers of copies of vectors to allow expression of GFP mRNAs with non-regulatory UTRs (positive control; "con"), or with reporters for each isoform-specific 3'UTR in combination with each 5'UTR. Translational efficiencies were determined as described above and are presented relative to positive controls (Fig 5). 3'UTRs had potent and differential influences on translational efficiencies. The ER $\beta 1$ 3'UTR had little influence on the translational efficiencies specified by the 5'UTRs in either cell line (compare lanes 2 with 1,8 with 7 and 14 with 13). In contrast, the two alternative ER $\beta 2$ 3'UTRs had markedly different effects. $\beta 2$-long had little influence on the translational efficiencies specified by 5 'UTRs (compare lanes 4 with 1, 10 with 7 and 16 with 13). However, $\beta 2$-short induced dramatic and, in the case of MCF7 cells, total derepression of the translation inhibition specified by 5'UTRa (compare lane 3 with $1 ; \mathrm{p}<0.0001$ in both cell lines), while having relatively little influence when paired with UTRc or UTR-E1 (compare lanes 9 with 7 and 15 with 13). ER 35 3'UTRs had smaller influences on translational efficiencies. $\beta 5 \mathrm{MCF}$ induced de-repression of the inhibitory influence of both UTRc and UTRE1 (compare lane 11 and 7; 13 with $8 ; \mathrm{p}<0.02$ in all cases), while $\beta 5 \mathrm{HB}$ had little influence except for inducing strong translation when paired with UTR-E1 (compare lane 18 with 13) in only HB2 cells (notably, these are the cells from which this 3'UTR was cloned). We concluded that cross-talk between 5' and 3'UTRs had profound influences on the translational efficiency of transcripts for individual ER $\beta$ isoforms, with length of $3^{\prime}$ UTRs - as defined by differential use of polyadenylation sites - being a critical factor in determining the outcome.

\section{7ß-estradiol (E2) modifies ERß transcription and translation}

E2 influences downstream effects of estrogen receptors by binding to and modifying their activity as transcription factors. It has also been reported that E2 impacts on expression of ER $\beta$ itself at both transcriptional [22, 23] and translational levels [24] by largely unknown mechanisms. We next investigated the influence of E2 on ER $\beta$ transcription and translation using our reporters for both these regulatory stages. Cells were transfected with equal copy numbers of either luciferase reporters containing different ER $\beta$ promoters (as Fig 2) or GFP reporters containing isoform-specific UTR pairings (as Fig 5) for analysis of influences on transcription and translation respectively. Cells were then treated with vehicle or E2 for $24 \mathrm{~h}$ and were analysed for luciferase activity or translational efficiency of GFP transcripts. In terms of transcriptional activity, E2 caused a 1.6 fold activation of the $0 \mathrm{~K}$ promoter in MCF7 cells $(p=0.001$ ) but not in $\mathrm{HB} 2$ cells (Fig 6A), while having no significant effect on promoters $0 \mathrm{~N}$ or E1 (Fig 6B and C). Effects of E2 on translational efficiencies specified by the various UTR pairings were more complex. Translation of transcripts containing the ER $\beta 1$ 3'UTR were increased by E2 when combined with UTRc or UTR-E1 (Fig 7A; $p=0.008$ and $p=0.03$ respectively). ER 32 3'UTRs (both short and long) specified an E2-dependent decrease in translational efficiency when paired with 5'UTRa (Fig 7B; both $\mathrm{p}=0.003$ ), but an increase when paired with UTR-E1 $(p=0.01$ and $p=0.049)$. ER $\beta 5$ 3'UTRs also specified differential responses to $E 2$, determining both decreases $(\beta 5$ MCF paired with UTR-E1; $p=0.005)$ and increases in translation ( $\beta 5$ HB paired with UTRc; $p=0.002$ ). We concluded that E2 has only relatively weak influences on ER $\beta$ transcription and translation. However, as these influences act differentially on the promoters and on the isoform-specific UTR pairings, these weak influences cumulatively have potential to modify the balance of ER $\beta$ isoforms. 


\section{DISCUSSION}

It is well established that the ER $\beta$ gene has two promoters allowing expression of full-length transcripts, promoters $0 \mathrm{~K}$ and $0 \mathrm{~N}$ [20]. Messages transcribed from each promoter have different 5'UTRs, termed UTRa and UTRc [16] (Fig 1B). However, full-length ER $\beta$ transcripts containing neither of these UTRs have been reported [25] and the presence of an additional promoter, giving rise to further alternative 5'UTRs, has long been suspected [26]. We have identified an additional 5'UTR, UTR-E1, and the corresponding novel promoter region, promoter E1 (Figs 1 and 2). A number of previous studies have attempted to assess the relative transcriptional strengths and thereby importance of promoters $0 \mathrm{~K}$ and $0 \mathrm{~N}$ [27, 28]. However, our previous data, demonstrating that ER $\beta$ 5'UTRs determine differential and cell-type specific translational inhibition [16], cast doubt on these assessments. Reporter assays including exonic sequences would combine translational differences with the expected measure of transcription. We have, therefore, investigated promoter activities of only sequences immediately upstream of the exons thereby avoiding translational regulatory motifs (although risking failure to include important transcription factor binding sites within the exons), and have analysed translational regulation separately. We find promoter $0 \mathrm{~N}$ to be the most active in both cell lines tested, although promoters E1 and $0 \mathrm{~K}$ are also active (Fig 2). Methylation of $\mathrm{CpG}$ islands within promoter $0 \mathrm{~N}$ has been reported in cancers, including those of the breast [29], ovary [30] and prostate [28], and this is thought to be responsible for down-regulation of some forms of ER $\beta$ during carcinogenesis [9, $10,31]$. In this context, it is interesting to note the presence of a $\mathrm{CpG}$ island within promoter $\mathrm{E} 1$ (from -2180 to -1878 with respect to the translational start); our preliminary data suggest that these sequences are also commonly methylated in breast cancers [32]. It is also interesting that the relative activities of the promoters as determined by luciferase assay (Figure 2) do not correlate with the expression of the 5'UTRs derived from the endogenous promoters (Figure 1C). This may relate to the inhibitory methylation of endogenous promoters, or to the influence of transcription factors binding outside the sequence included in the reporter constructs.

We previously demonstrated that ER $\beta$ expression is regulated at the level of translation, with UTRa and UTRc having potent and differential influences [16]. When these analyses were extended to include UTR-E1, we found that UTR-E1 allowed the most efficient translation of the ER $\beta$ 5'UTRs (Fig 3). Therefore, transcripts from promoter E1, which contain UTR-E1, are likely to contribute disproportionally to ER $\beta$ protein levels. Moreover, a key finding here is that translational regulation acts differentially on transcripts for the ER $\beta$ isoforms 1,2 and 5 . These transcripts are derived from differential 3' splicing [4], giving them isoform-specific 3'UTRs. We identified 3'UTR sequences for each isoform (Fig 4) and demonstrated that when combined with ER $\beta$ 5'UTRs these specified a wide range of translational efficiencies (Fig 5). Most striking was the ability of different ER $\beta 2$ 3'UTRs to define up to a 10 fold difference in translational efficiency when paired with UTRa. 5'UTRa inhibits translation on account of inefficient translational scanning induced by secondary structure and initiation at upstream open reading frames (uORFs) [16]. The shorter ER $\beta 2$ 3'UTR ( $\beta 2$-short) overcame this inhibitory influence, while the longer form ( $\beta 2$-long), which included the entire sequence of $\beta 2$-short, had little effect. Regulatory elements in both 5' and 3'UTRs have important roles in determining translational efficiencies, facilitated by transcript circularisation induced by interaction of poly(A)-binding protein binding at the 3 ' of transcripts with eIF4G at 5' ends $[33,34]$. $\beta 2$-short apparently interacted with 5'UTRa resulting in loss of inhibitory structures and/or uORF translation, while the same sequences were unable to interact in this way in the context of $\beta 2$-long. Therefore, 
differential use of polyadenylation sites, defining 3'UTR length, exerts translational control. It is interesting that faster-proliferating cells and cancer cells preferentially use proximal polyadenylation sites $[35,36]$; in the case of ER $\beta 2$, this would lead to increased translation that may explain how ER $\beta 2$ is up-regulated in breast cancer cells [10, 14] despite promoter methylation and down-regulation of ER $\beta$ transcripts [29, 37]. Shortening of 3'UTRs has been associated with increased protein expression for a number of cancer-related genes [36]. For these genes, this resulted from increased mRNA stability and/or loss of microRNA-mediated translational repression when compared to their longer 3'UTRs. For ER $\beta 2$, this is clearly not the case, since $\beta 2$-short dominantly de-repressed the influence of 5'UTRa, as opposed to merely lacking repressive 3' elements present in $\beta 2$-long. ER $\beta 5$ 3'UTRs also tended to specify relatively efficient translation, but in this case, only when modifying the influences of 5'UTRc or UTR-E1. In MCF7 cells, this was only apparent for the ER 35 3'UTR that was actually cloned from these cells. As for ER $\beta 2$, the data do not support a simple model of shorter 3'UTRs having fewer repressive elements since both lengths of ER $\beta 5$ 3'UTR acted to de-repress the influences of inhibitory 5'UTRs.

Finally, we investigated influences of the ER ligand E2 on transcriptional activity of ER $\beta$ promoters and translation specified by ER $\beta$ UTRs. We found E2 exerted relatively mild, although statistically significant, influences at multiple levels, with potential to change the balance of ER $\beta$ isoform expression. Unfortunately, we were unable to confirm whether this balance is altered since quantitative detection of endogenous ER $\beta$ protein isoforms in cell lines is unreliable with the antibodies available currently. First, we found E2 to induce transcription in MCF7 cells of only the $0 \mathrm{~K}$ promoter (Fig 6), a promoter we have previously shown to exhibit a relative preference for producing ER $\beta 1$ and 5 transcripts in this cell type [16]. Secondly, we found E2 to induce a wide range of changes in translational efficiencies of transcripts with ER $\beta$ UTR pairs (Fig 7); the overall effect in any cell type would be defined by the relative proportions of each UTR pairing within the total pool of transcripts for that isoform. E2 is known to modulate levels of microRNAs [38, 39], thus these constitute potential mediators of its influence on ER $\beta$ translational efficiencies. Indeed, we have found that miR-92 can regulate expression of ER $\beta 1$ acting at its 3'UTR [40]. However, we find that different UTR pairs respond differently, therefore we infer that accessibility of individual microRNA binding sites in the context of interactions between 5' and 3'UTRs would influence whether particular transcripts respond to changes in levels of any particular microRNA.

In conclusion, we have revealed novel mechanisms controlling ER $\beta$ expression that help explain the reported lack of concordance between ER $\beta$ mRNA and protein levels, and the differential expression of ER $\beta 1,2$ and 5, and give new insights into the regulation of estrogen receptor function. To the best of our knowledge, this is the first time cross-talk between multiple 5' and 3'UTRs has been implicated in the differential regulation of translation of different protein isoforms from one gene, demonstrating how the ER $\beta$ gene provides a model for study of complex gene regulatory pathways.

\section{FUNDING}

We thank Breast Cancer Campaign, the Breast Cancer Research Action Group, the US Department of Defense, and Yorkshire Cancer Research for funding. 


\section{REFERENCES}

1 Chen, G. G., Zeng, Q. and Tse, G. M. (2008) Estrogen and its receptors in cancer. Med Res Rev. 28, 954-974

2 O'Neill, P. A., Davies, M. P., Shaaban, A. M., Innes, H., Torevell, A., Sibson, D. R. and Foster, C. S. (2004) Wild-type oestrogen receptor beta (ERbeta1) mRNA and protein expression in Tamoxifen-treated post-menopausal breast cancers. Br. J. Cancer. 91, 1694-1702

3 Green, C. A., Peter, M. B., Speirs, V. and Shaaban, A. M. (2008) The potential role of ER beta isoforms in the clinical management of breast cancer. Histopathology. 53, 374-380

4 Moore, J. T., McKee, D. D., Slentz-Kesler, K., Moore, L. B., Jones, S. A., Horne, E. L., Su, J. L., Kliewer, S. A., Lehmann, J. M. and Willson, T. M. (1998) Cloning and characterization of human estrogen receptor beta isoforms. Biochem. Biophys. Res. Commun. 247, 75-78

5 Shaaban, A. M., Green, A. R., Karthik, S., Alizadeh, Y., Hughes, T. A., Harkins, L., Ellis, I. O., Robertson, J. F., Paish, E. C., Saunders, P. T., Groome, N. P. and Speirs, V. (2008) Nuclear and cytoplasmic expression of ERbeta1, ERbeta2, and ERbeta5 identifies distinct prognostic outcome for breast cancer patients. Clin Cancer Res. 14, 5228-5235

6 Honma, N., Horii, R., Iwase, T., Saji, S., Younes, M., Takubo, K., Matsuura, M., Ito, Y., Akiyama, F. and Sakamoto, G. (2008) Clinical importance of estrogen receptor-beta evaluation in breast cancer patients treated with adjuvant tamoxifen therapy. J Clin Oncol. 26, 3727-3734

7 Novelli, F., Milella, M., Melucci, E., Di Benedetto, A., Sperduti, I., Perrone-Donnorso, R., Perracchio, L., Venturo, I., Nistico, C., Fabi, A., Buglioni, S., Natali, P. G. and Mottolese, M. (2008) A divergent role for estrogen receptor-beta in node-positive and node-negative breast cancer classified according to molecular subtypes: an observational prospective study. Breast Cancer Res. 10, R74

8 Vinayagam, R., Sibson, D. R., Holcombe, C., Aachi, V. and Davies, M. P. (2007) Association of oestrogen receptor beta 2 (ER beta 2/ER beta cx) with outcome of adjuvant endocrine treatment for primary breast cancer--a retrospective study. BMC Cancer. 7, 131

9 Skliris, G. P., Munot, K., Bell, S. M., Carder, P. J., Lane, S., Horgan, K., Lansdown, M. R., Parkes, A. T., Hanby, A. M., Markham, A. F. and Speirs, V. (2003) Reduced expression of oestrogen receptor beta in invasive breast cancer and its re-expression using DNA methyl transferase inhibitors in a cell line model. J Pathol. 201, 213-220

10 Shaaban, A. M., O'Neill, P. A., Davies, M. P., Sibson, R., West, C. R., Smith, P. H. and Foster, C. S. (2003) Declining estrogen receptor-beta expression defines malignant progression of human breast neoplasia. Am J Surg Pathol. 27, 1502-1512

11 Bardin, A., Boulle, N., Lazennec, G., Vignon, F. and Pujol, P. (2004) Loss of ERbeta expression as a common step in estrogen-dependent tumor progression. Endocr Relat Cancer. 11, 537-551

12 Lazennec, G. (2006) Estrogen receptor beta, a possible tumor suppressor involved in ovarian carcinogenesis. Cancer Lett. 231, 151-157

13 Galluzzo, P., Caiazza, F., Moreno, S. and Marino, M. (2007) Role of ERbeta palmitoylation in the inhibition of human colon cancer cell proliferation. Endocr Relat Cancer. 14, 153-167

14 Esslimani-Sahla, M., Kramar, A., Simony-Lafontaine, J., Warner, M., Gustafsson, J. A. and Rochefort, H. (2005) Increased estrogen receptor betacx expression during mammary carcinogenesis. Clin Cancer Res. 11, 3170-3174

15 Park, B. W., Kim, K. S., Heo, M. K., Yang, W. I., Kim, S. I., Kim, J. H., Kim, G. E. and Lee, K. S. (2006) The changes of estrogen receptor-beta variants expression in breast 
carcinogenesis: Decrease of estrogen receptor-beta2 expression is the key event in breast cancer development. J Surg Oncol. 93, 504-510

16 Smith, L., Brannan, R. A., Hanby, A. M., Shaaban, A. M., Verghese, E. T., Peter, M., Pollock, S., Satheesha, S., Szynkiewicz, M., Speirs, V. and Hughes, T. A. (2009) Differential regulation of estrogen receptor $\beta$ isoforms by 5 , untranslated regions in cancer. Journal of Cellular and Molecular Medicine, E-pub ahead of print

17 Hughes, T. A. and Brady, H. J. M. (2005) Cross-talk between pRb/E2F and Wnt/bcatenin pathways: E2F1 induces axin2 leading to repression of Wnt signalling and to increased cell death. Exp. Cell Res. 303, 32-46

18 Akamine, R., Yamamoto, T., Watanabe, M., Yamazaki, N., Kataoka, M., Ishikawa, M., Ooie, T., Baba, Y. and Shinohara, Y. (2007) Usefulness of the 5' region of the cDNA encoding acidic ribosomal phosphoprotein P0 conserved among rats, mice, and humans as a standard probe for gene expression analysis in different tissues and animal species. J Biochem Biophys Methods. 70, 481-486

19 Zuker, M. (2003) Mfold web server for nucleic acid folding and hydrization prediction. Nuc. Acids Res. 31, 3406-3415

20 Hirata, S., Shoda, T., Kato, J. and Hoshi, K. (2001) The multiple untranslated first exons system of the human estrogen receptor beta (ER beta) gene. J Steroid Biochem Mol Biol. 78, 3340

21 Hughes, T. A. and Brady, H. J. M. (2005) Expression of axin2 is regulated by the alternative 5' untranslated regions of its mRNA.J. Biol. Chem. 280, 8581-8588

22 Catanuto, P., Doublier, S., Lupia, E., Fornoni, A., Berho, M., Karl, M., Striker, G. E., Xia, X. and Elliot, S. (2009) 17 beta-estradiol and tamoxifen upregulate estrogen receptor beta expression and control podocyte signaling pathways in a model of type 2 diabetes. Kidney Int. 75, 1194-1201

23 Montanaro, D., Maggiolini, M., Recchia, A. G., Sirianni, R., Aquila, S., Barzon, L., Fallo, F., Ando, S. and Pezzi, V. (2005) Antiestrogens upregulate estrogen receptor beta expression and inhibit adrenocortical H295R cell proliferation. J Mol Endocrinol. 35, 245-256

24 Caiazza, F., Galluzzo, P., Lorenzetti, S. and Marino, M. (2007) 17Beta-estradiol induces ERbeta up-regulation via p38/MAPK activation in colon cancer cells. Biochem. Biophys. Res. Commun. 359, 102-107

25 Ogawa, S., Inoue, S., Watanabe, T., Hiroi, H., Orimo, A., Hosoi, T., Ouchi, Y. and Muramatsu, M. (1998) The complete primary structure of human estrogen receptor beta (hER beta) and its heterodimerization with ER alpha in vivo and in vitro. Biochem. Biophys. Res. Commun. 243, 122-126

26 Zhao, C., Dahlman-Wright, K. and Gustafsson, J. A. (2008) Estrogen receptor beta: an overview and update. Nucl Recept Signal. 6, e003

27 Xue, Q., Lin, Z., Cheng, Y. H., Huang, C. C., Marsh, E., Yin, P., Milad, M. P., Confino, E., Reierstad, S., Innes, J. and Bulun, S. E. (2007) Promoter methylation regulates estrogen receptor 2 in human endometrium and endometriosis. Biol Reprod. 77, 681-687

28 Zhang, X., Leung, Y. K. and Ho, S. M. (2007) AP-2 regulates the transcription of estrogen receptor (ER)-beta by acting through a methylation hotspot of the $0 \mathrm{~N}$ promoter in prostate cancer cells. Oncogene. 26, 7346-7354

29 Zhao, C., Lam, E. W., Sunters, A., Enmark, E., De Bella, M. T., Coombes, R. C., Gustafsson, J. A. and Dahlman-Wright, K. (2003) Expression of estrogen receptor beta isoforms 
in normal breast epithelial cells and breast cancer: regulation by methylation. Oncogene. 22, 7600-7606

30 Suzuki, F., Akahira, J. I., Miura, I., Suzuki, T., Ito, K., Hayashi, S. I., Sasano, H. and Yaegashi, N. (2008) Loss of estrogen receptor beta isoform expression and its correlation with aberrant DNA methylation of the 5'-untranslated region in human epithelial ovarian carcinoma. Cancer Sci. 99, 2365-2372

31 Roger, P., Sahla, M. E., Makela, S., Gustafsson, J. A., Baldet, P. and Rochefort, H. (2001) Decreased expression of estrogen receptor beta protein in proliferative preinvasive mammary tumors. Cancer Res. 61, 2537-2541

32 Al-Nakhle, H. H., Smith, L., Hughes, T. A., Cummings, M., Hanby, A. M., Shaaban, A. M., Burns, P. A. and Speirs, V. (2009) Methylation Status of Promoters 0K, 0N and a Newly Identified Promoter Regulate ER beta 1 Expression in Breast Cancer. Cancer Res. 69, 585S-586S 33 Cheng, S. and Gallie, D. R. (2007) eIF4G, eIFiso4G, and eIF4B bind the poly(A)-binding protein through overlapping sites within the RNA recognition motif domains. J. Biol. Chem. 282, 25247-25258

34 Komarova, A. V., Brocard, M. and Kean, K. M. (2006) The case for mRNA 5' and 3' end cross talk during translation in a eukaryotic cell. Prog Nucleic Acid Res Mol Biol. 81, 331-367

35 Sandberg, R., Neilson, J. R., Sarma, A., Sharp, P. A. and Burge, C. B. (2008) Proliferating cells express mRNAs with shortened 3' untranslated regions and fewer microRNA target sites. Science. 320, 1643-1647

36 Mayr, C. and Bartel, D. P. (2009) Widespread shortening of 3'UTRs by alternative cleavage and polyadenylation activates oncogenes in cancer cells. Cell. 138, 673-684

37 Rody, A., Holtrich, U., Solbach, C., Kourtis, K., von Minckwitz, G., Engels, K., Kissler, S., Gatje, R., Karn, T. and Kaufmann, M. (2005) Methylation of estrogen receptor beta promoter correlates with loss of ER-beta expression in mammary carcinoma and is an early indication marker in premalignant lesions. Endocr Relat Cancer. 12, 903-916

38 Bhat-Nakshatri, P., Wang, G., Collins, N. R., Thomson, M. J., Geistlinger, T. R., Carroll, J. S., Brown, M., Hammond, S., Srour, E. F., Liu, Y. and Nakshatri, H. (2009) Estradiolregulated microRNAs control estradiol response in breast cancer cells. Nuc. Acids Res. 37, 4850-4861

39 Maillot, G., Lacroix-Triki, M., Pierredon, S., Gratadou, L., Schmidt, S., Benes, V., Roche, H., Dalenc, F., Auboeuf, D., Millevoi, S. and Vagner, S. (2009) Widespread estrogendependent repression of micrornas involved in breast tumor cell growth. Cancer Res. 69, 83328340

40 Al-Nakhle, H. H., Burns, P. A., Cummings, M., Hanby, A. M., Hughes, T. A., Satheesha, S., Shaaban, A. M., Smith, L. and Speirs, V. (2009) miR-92 Is a Novel Regulator of ER beta 1 Expression in Breast Cancer. Cancer Res. 69, 753S-754S 


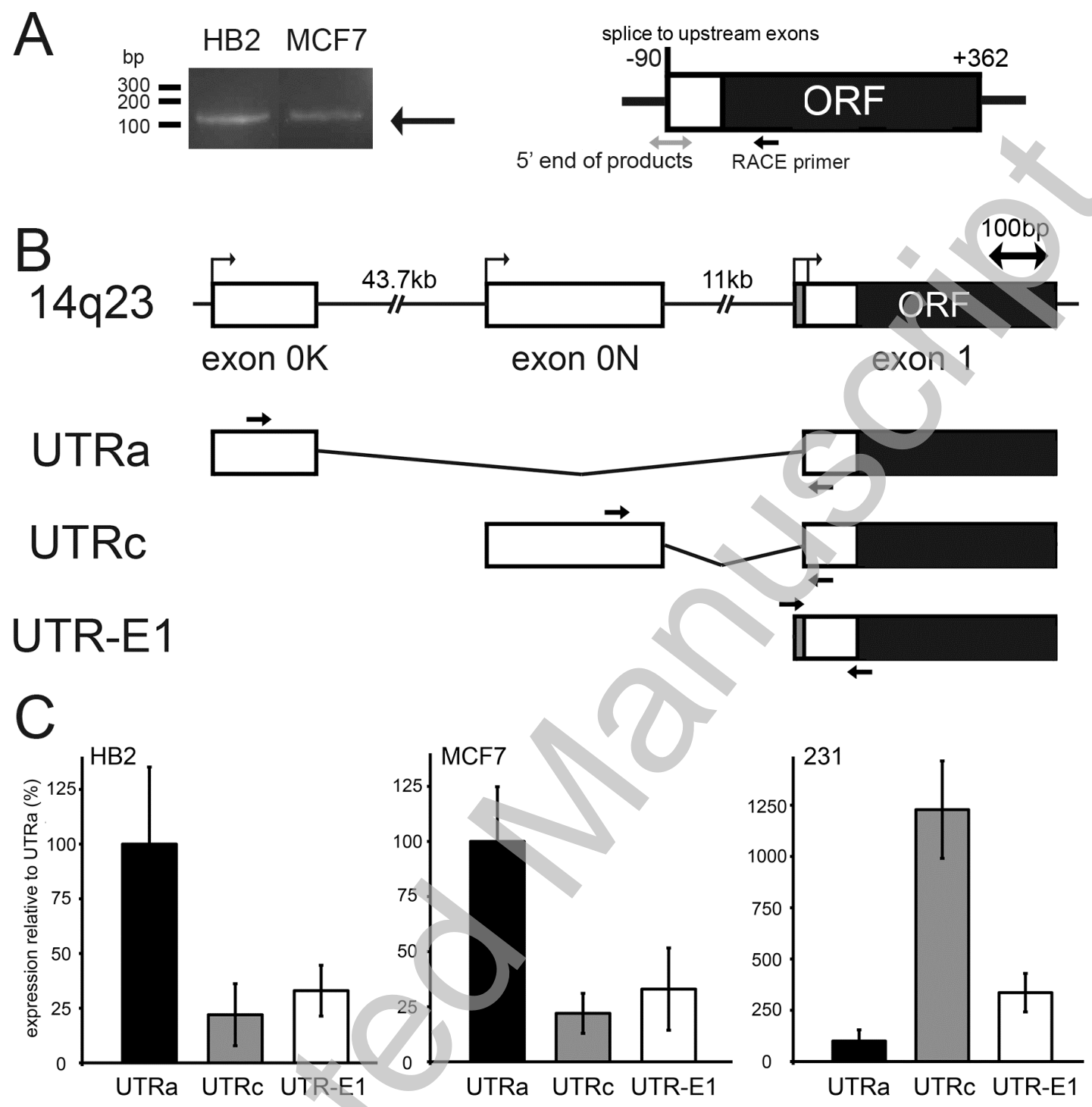

Figure 1 A novel ER $\beta$ 5'UTR. A) 5'RACE analyses of ER $\beta$ transcripts were performed; products representing a previously unidentified 5'UTR containing sequence from or immediately upstream of exon 1 were amplified. Products from two breast cell lines are shown along with a diagram representing ER $\beta$ exon 1 (genomic locations with respect to the translational start are indicated). B) Alignment of the 5' end of the human ER $\beta$ gene with mRNAs containing either UTRa, UTRc or the novel UTR-E1. Coding regions (black boxes), transcriptional start sites (black arrows) and primers used for the qPCR analysis of each 5'UTR (black arrows) are shown. The grey box indicates sequence included in a subset of transcripts with UTR-E1. C) Relative expression of UTRa, UTRc and UTR-E1 in breast cell lines was examined by qPCR; all three cell lines examined expressed UTR-E1. 

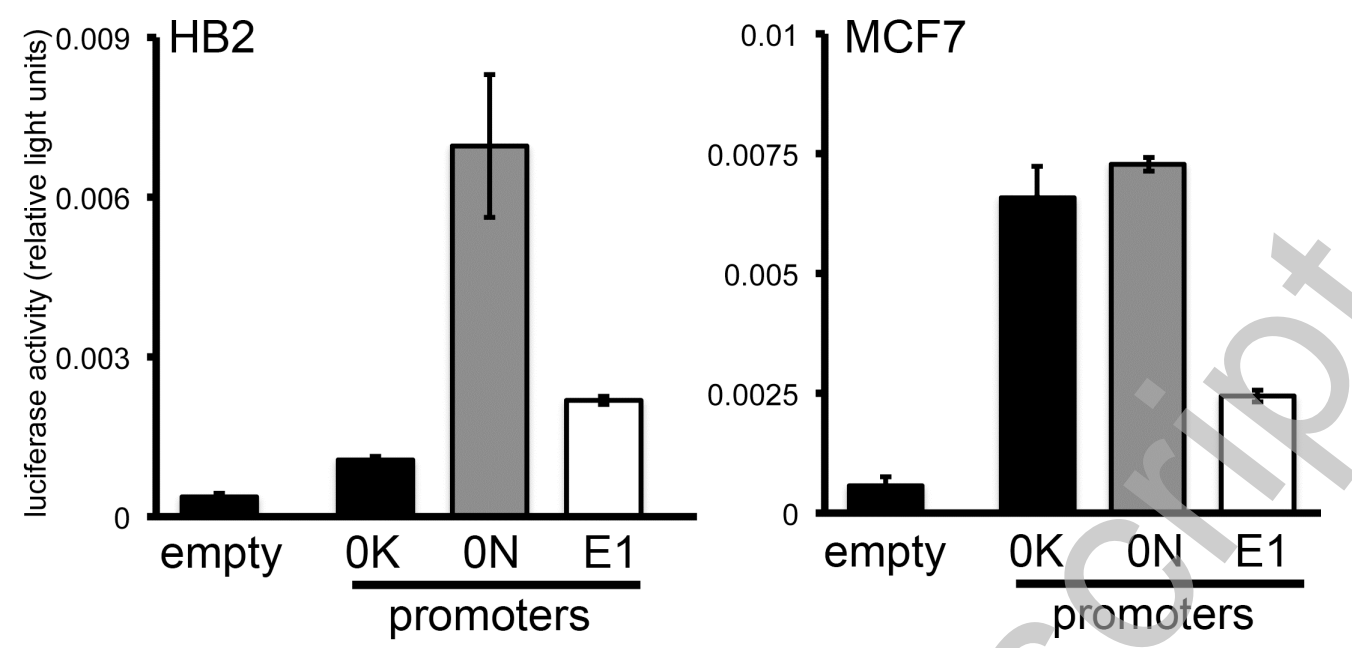

Figure $2 \mathrm{~A}$ third ER $\beta$ promoter shows activity in breast cell lines. $\sim 2 \mathrm{~kb}$ of genomic DNA directly upstream of exons $0 \mathrm{~K}, 0 \mathrm{~N}$ or $\mathrm{E} 1$ was cloned into luciferase reporter vectors. $\mathrm{HB} 2$ and MCF7 cell lines were transiently transfected with equal copy numbers of luciferase reporter lacking additional promoter sequences (empty) or containing promoter sequences as shown, and luciferase assays were performed after $24 \mathrm{~h}$. A minimum of two independent experiments were performed and within each experiment three technical replicates were included. Error bars show the standard deviation of technical triplicates within a representative experiment.

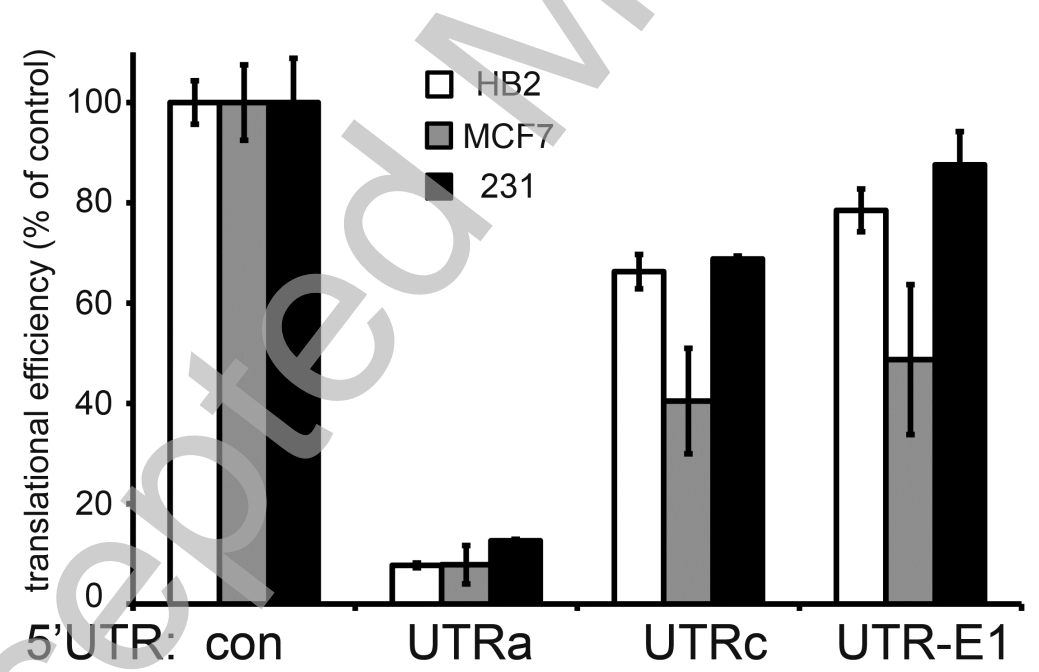

Figure 3 5'UTRs regulate ER $\beta$ translational efficiency. Reporters were constructed to express mRNAs containing the GFP reading frame preceded by different 5'UTRs: a control sequence lacking regulatory motifs, UTRa, UTRc or UTR-E1. Cell lines were transiently transfected with equal copy numbers of either control or experimental constructs. GFP protein and mRNA were quantified by flow-cytometry and real-time PCR respectively. Translational efficiency (protein synthesised per unit mRNA) is presented relative to the GFP control that lacks a specialised 5'UTR. A minimum of two independent experiments were performed and within each experiment three technical replicates were included. Error bars show the standard deviation of technical triplicates within a representative experiment. 


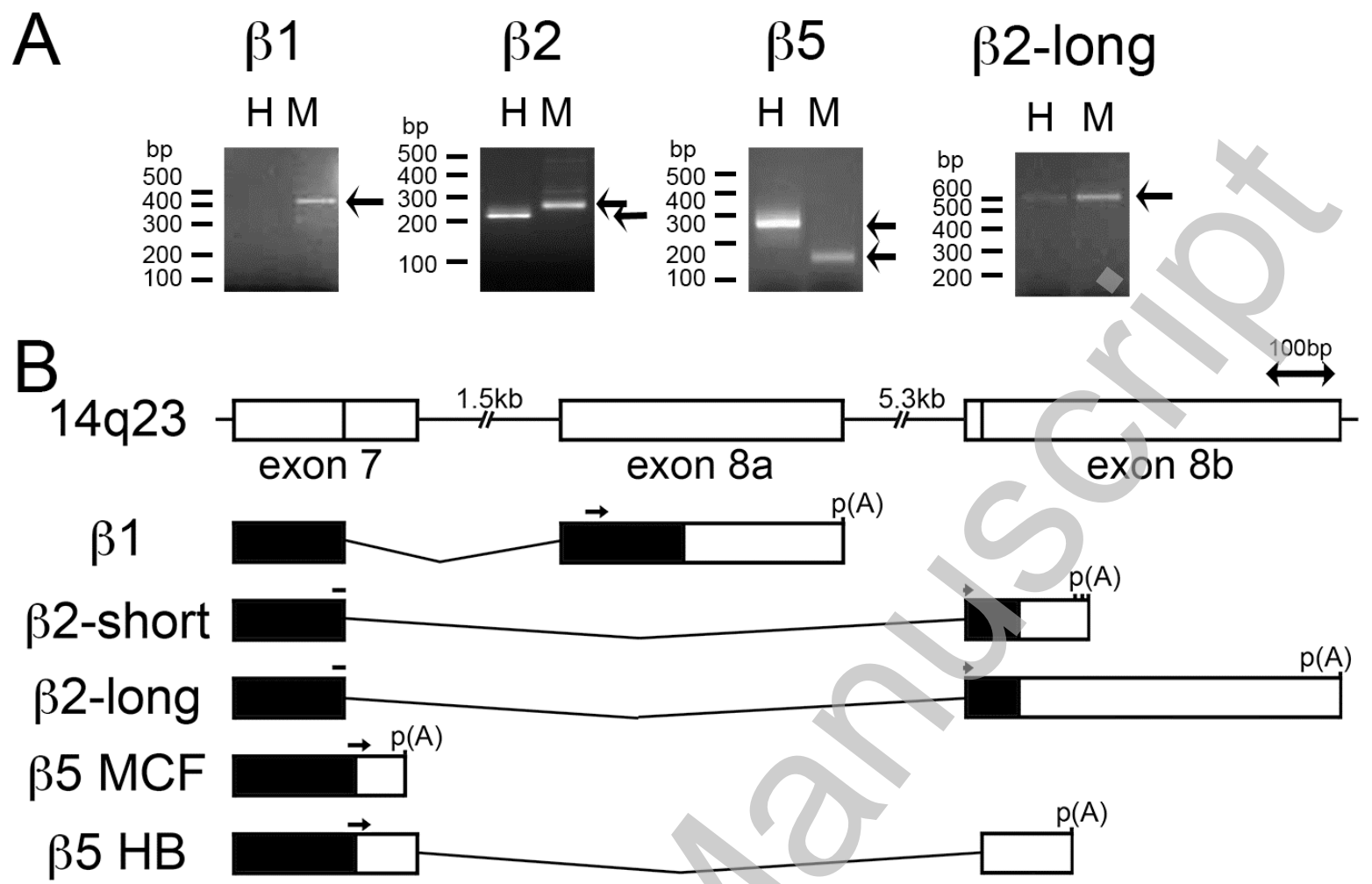

Figure 4 Differential splicing confers multiple and different 3'UTRs on transcripts for each ER $\beta$ isoform. A) 3'RACE analyses for each ER $\beta$ isoform were performed on the breast cell lines HB2 $(\mathrm{H})$ and MCF7 $(\mathrm{M})$. Products were identified and sequenced. PCR analysis was also performed using primers specific for an ER $\beta 23^{\prime} \mathrm{UTR}$ that is represented within Genbank ( $\beta 2$-long; right hand panel). B) Alignment of the 3' end of the human ER $\beta$ gene with mRNAs for each isoform containing the 3'UTRs (open boxes) identified. Coding regions (black boxes) and the location of polyadenylation site $(\mathrm{p}(\mathrm{A}))$ and primers used for amplification of products in A (black arrows) are shown. 

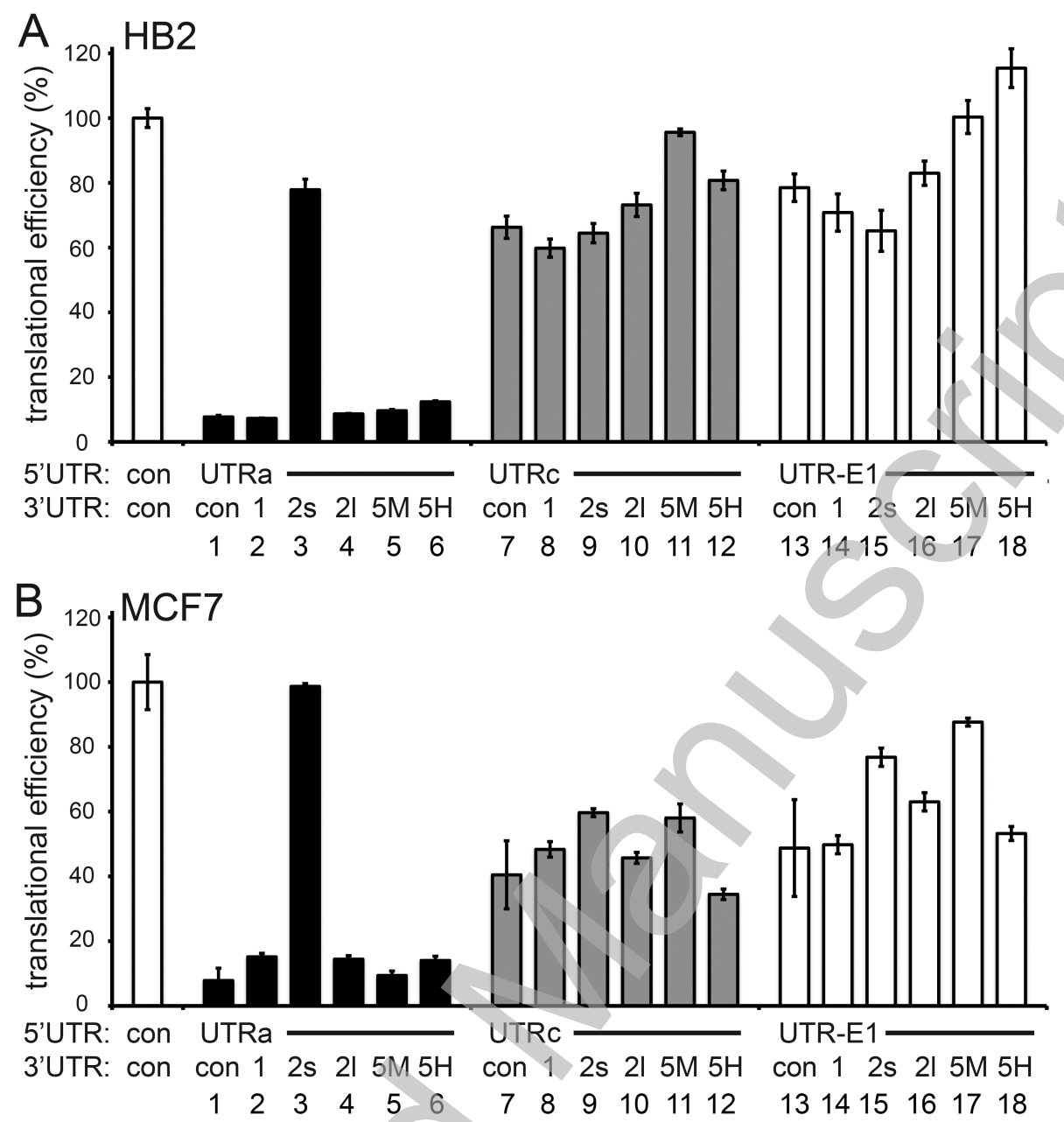

Figure 5 Isoform-specific 3'UTRs define different translational efficiencies in conjunction with 5'UTRs. 3'UTRs were cloned downstream of the GFP reading frame in each 5'UTR GFP reporter construct. HB2 (A) and MCF7 (B) cells were transiently transfected with reporter constructs and translational efficiencies determined relative to the GFP control. A minimum of two independent experiments were performed and within each experiment three technical replicates were included. Error bars show the standard deviation of technical triplicates within a representative experiment. 

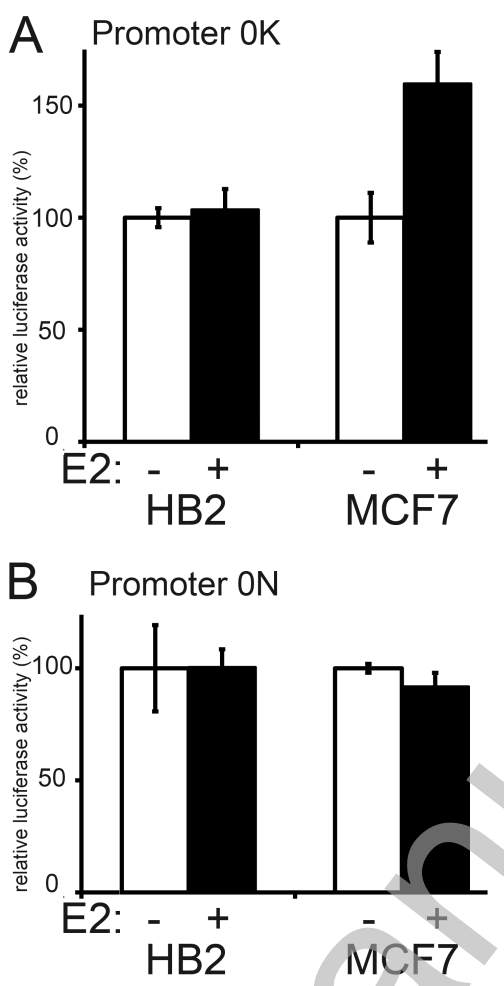

C Promoter E1

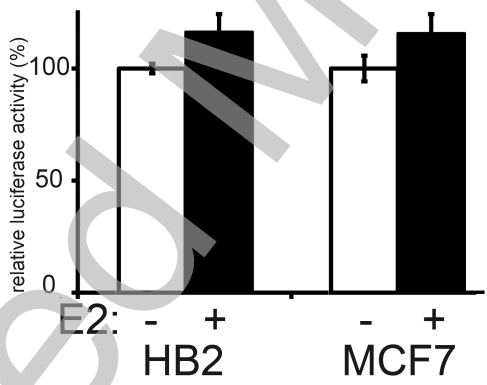

Figure 6 E2 modifies ER $\beta$ transcription. HB2 and MCF7 cells were transfected with equal copy numbers of luciferase reporters containing different ER $\beta$ promoters (as Fig 2). Cells were treated with vehicle (white bars) or 10nM E2 (black bars) for $24 \mathrm{~h}$ and luciferase assays were performed. E2 caused an increase in the activity of 0K promoter in MCF7 cells but not in HB2 cells (A), while having no significant effect on promoters $0 \mathrm{~N}$ or $\mathrm{E} 1 \mathrm{~B}$ and $\mathrm{C}$ ). A minimum of two independent experiments were performed and within each experiment three technical replicates were included. Error bars show the standard deviation of technical triplicates within a representative experiment. 



Figure 7 E2 modifies ER $\beta$ translation. MCF7 cells were transfected with equal copy numbers of GFP reporters containing UTR pairings (as Fig 5). Cells were treated with vehicle (white bars) or 10nM E2 (black bars) for $24 \mathrm{~h}$ and translational efficiency of GFP transcripts was determined. A minimum of two independent experiments were performed and within each experiment three technical replicates were included. Error bars show the standard deviation of technical triplicates within a representative experiment. 